\title{
Male nutritional history affects female fecundity in a male-dimorphic mite: Evidence for a nuptial gift?
}

\author{
T. P. G. van den Beuken ${ }^{1}$ I. M. Smallegange ${ }^{1}$
}

Received: 4 December 2017 / Accepted: 18 May 2018 / Published online: 24 May 2018

(C) The Author(s) 2018

\begin{abstract}
In male-dimorphic species, males are often either armoured 'majors' that can monopolise access to females, or unarmoured and defenceless 'minors' that cannot. However, majors, unlike minors, have to spend energy to maintain their weaponry. Like-for-like, this could mean that minors have relatively more energy available to increase their reproductive output through e.g. sperm competition, or the transference of nutrients by means of a nuptial gift. Such a fitness advantage to minors could therefore contribute to explaining the coexistence of both morphs in single populations. We tested if food-deprived females of the male-dimorphic bulb mite Rhizoglyphus robini produced more eggs when mated to a minor or to a major male, and whether egg production depended on whether their mates were starved or fed prior to mating. We found no effect of male morph on female fecundity, but females did produce more eggs when mated to previously fed males. We also found that females increased in mass, but males decreased in mass over the course of the experiment. From these observations we infer that fed males are able to transfer nutrients, a nuptial gift, to their mate. This is the first observation to suggest nuptial gift transfer in mites. Though males of both morphs appeared able to produce nuptial gifts; other factors, like habitat complexity, should be considered to identify fitness benefits of minor over major males to understand why the two morphs coexist.
\end{abstract}

Keywords Alternative reproductive tactics - ARTs - ARPs - Diet · Nutritional state · Seminal gifts

Electronic supplementary material The online version of this article (https://doi.org/10.1007/s1068 2-018-9940-9) contains supplementary material, which is available to authorized users.

T. P. G. van den Beuken

t.p.g.vandenbeuken@outlook.com

1 Department of Evolutionary and Population Biology, Institute for Biodiversity and Ecosystem Dynamics, University of Amsterdam, Science Park 904, PO Box 94240, 1090 GE Amsterdam, The Netherlands 


\section{Introduction}

Alternative reproductive phenotypes (ARPs) represent discontinuous variations in behaviour, morphology or physiology between members of the same sex related to intrasexual competition over access to mates (Oliveira et al. 2008). ARPs have evolved across a wide range of animal taxa (Gross 1996; Oliveira et al. 2008) including salmon (Morasse et al. 2014), dung beetles (Simmons et al. 1999), howler monkeys (Dunn et al. 2015), side-blotched lizards (Sinervo et al. 2001), ruffs (Jukema and Piersma 2006) and squid (Iwata et al. 2011). Because the evolution of ARPs is hypothesized to be the result of strong sexual selection, and sexual selection is typically stronger in males, ARPs can be expected to evolve more frequently in males than in females (Taborsky et al. 2008).

Many male ARPs comprise two morphs: 'majors' and 'minors' (Oliveira et al. 2008), the names by which these two morphs are referred to vary across taxa (Taborsky 1997). Majors typically invest in morphological structures that act as weaponry when competing with conspecific males e.g. to gain access to or monopolize females (Oliveira et al. 2008). A male is more likely to become a major if it has accumulated sufficient resources during ontogeny to support the development of weaponry (Roff 1996). Males that have not accumulated sufficient resources develop into minors (Roff 1996). Minors generally have lower fitness than fighters and have consequently long been considered to 'try to make the best of a bad job' (Dawkins 1980; Eberhard 1982; Smith 1982; Oliveira et al. 2008). However, we now know that minors can adopt alternative strategies to increase their reproductive success by investing into qualities that help them to be more successful during copulations. For example, minors of some species produce more sperm cells than majors, making minors more likely to win in a sperm competition (Kvarnemo et al. 2010; Smith and Ryan 2010; Young et al. 2013). Minors of other species influence the outcome of sperm competitions by means of producing sperm that swims faster or contains more energy (Locatello et al. 2007). Males in some male-monomorphic species are known to invest into agents that increase their mate's fecundity, for instance nuptial gifts, which are compounds beneficial to the female through their nutritional value such as glandular products (Vahed 1998) prey, (part of) the male body (Eggert and Sakaluk 1994; Prenter et al. 2006) or seminal gifts (Bownes and Partridge 1987; Markow et al. 1990; Eisner et al. 1996; Edvardsson 2007; Gwynne 2008). However, whether or not minor males invest into nuptial gifts to increase their mate's reproductive success is, to our knowledge, unknown.

Minors are likely able to invest more resources into reproductive traits than majors because the major's weaponry can be energetically costly. This is because major's physical weaponry often makes locomotion less efficient (e.g. Basolo and Alcaraz 2003; Allen and Levinton 2007; Goyens et al. 2015) and because size-for-size, majors likely suffer higher maintenance costs than minors as they have a larger body volume on account of their weaponry (Kooijman and Metz 1983; Parker 1983; Emlen 2008; Stuglik et al. 2014). When compounds that increase female fecundity are costly to produce (Cordero 1995; Vahed 1998), having weaponry leads to a lower maximum investment in compounds to increase female fecundity. If energy budgets are otherwise comparable between majors and minors, it follows that minors could invest more energy in compounds transferred during mating to increase the female's fecundity. Such fitness advantages of minors over majors could help explain why these two morphs coexist in single populations.

Here, we investigated if minors have a fitness advantage compared to majors in the number of eggs their mates produce. We tested our hypothesis in the male-dimorphic bulb mite Rhizoglyphus robini. Bulb mite majors are referred to as 'fighters' and have sharply 
terminated legs that can be used to kill competitors; minors, which are called 'scramblers', do not have this weaponry (Radwan et al. 2000). Whether a male matures as a fighter or a scrambler is strongly dependent on its energy reserves, i.e. its body size, during its final quiescent stage: relatively large tritonymphs (final larval stage) are more likely to become fighters than small tritonymphs (Smallegange 2011). The few fitness benefits for scramblers that have been found are a shorter maturation time, allowing scramblers to start mating earlier in life than fighters from the same age cohort (Smallegange 2011), and a longer life span, although this benefit could decrease with age as fecundity decreases with age (Radwan and Bogacz 2000). Unlike minors in several other species (e.g. Simmons et al. 2007; Young et al. 2013), there is no evidence that scramblers outcompete fighters in direct sperm competition (Radwan 1997; Radwan and Bogacz 2000).

To test if females mated to scramblers have a higher fecundity than when mated to fighters, we conducted an experiment in which we recorded the fecundity of virgin females that were mated singly to either a virgin fighter or scrambler. We hypothesized that differences between the reproductive output of scramblers and fighters are the result of differences in energy budgets directly available for investment into reproduction. Fighters need to maintain their energetically expensive leg structures that they use in male-male competition; scramblers do not have such structures so that they could have more energy available to invest directly into reproduction. If this difference exists, it will be most evident when males are starved: for example, in Drosophila grimshawi, body condition and testes size only have a negative correlation under starved conditions (Droney 1998; also see Jia et al. 2000; Burris and Dam 2015). Therefore, in our experiment, we starved half of all males from the adult stage onwards. Because males may need a minimal amount of resources in order to be able to invest in reproduction at all, we fed the other half of the males for a single day, immediately after maturation and 1 day before mating. All females were starved to increase their sensitivity to nutritional value of sperm (also see Markow et al. 1990; Immonen et al. 2009). We regularly scored the mass of both males and females and the number of eggs laid by females, as (1) we expect males to lose mass through investment into reproductive output (e.g. nuptial gifts), and (2), after mating, the females to increase in mass and/or fecundity.

\section{Materials and methods}

\section{Life cycle of the bulb mite}

The bulb mite $R$. robini Claparède (Acari: Acaridae) lives in the soil and is a cosmopolitan pest of roots, bulbs and tubers of a large number of plants, including agriculturally important crops such as onions, lilies and garlic (Díaz et al. 2000). The life cycle of the bulb mite consists of six stages: egg, larva, protonymph, deutonymph (facultative dispersal stage), tritonymph and adult (Díaz et al. 2000). Between each stage, the mites go through a quiescent (sessile) moulting stage. The mites' sizes range from $100 \mu \mathrm{m}$ for eggs to $1000 \mu \mathrm{m}$ for adult females (Smallegange 2011). Depending on various environmental conditions, such as temperature and food quality, the time to develop from egg to adult takes 11-40 days (Smallegange 2011) and the adult stage lasts for 31-130 days (Gerson et al. 1983; Díaz et al. 2000). Adult males are dimorphic; they are either fighters or scramblers (Radwan 1995). 


\section{Maintenance of mites}

Stock cultures were kept at the University of Amsterdam (the Netherlands) and have been obtained in 2010 from storage rooms of flower bulbs in North Holland, The Netherlands. Henceforth, they were maintained in the laboratory and fed on yeast. Stock cultures were each kept in small plastic containers $(1 \times \mathrm{w} \times \mathrm{h}: 4 \times 4 \times 2.5 \mathrm{~cm})$ on a near water-saturated substratum of plaster of Paris covered with a layer of yeast granules (Bruggeman instant yeast). A square hole was cut in the lid of the plastic containers to allow air flow and evaporation of water, and a fine mesh covered the hole to avoid mites escaping. To prevent a build-up of detritus in the containers, about a third of the substratum was scraped away from each container twice a week, thereby removing food, detritus and about a third of the mite population. Hereafter, yeast was replenished and drops of water added.

During the experiment, mites were kept individually or in pairs (see Experimental setup $)$ in plastic tubes $(\mathrm{h} \times \mathrm{d}: 50 \times 16 \mathrm{~mm})$ filled with a layer of plaster of Paris darkened with powdered charcoal (to increase the contrast between the mites and the substrate). This plaster mixture was nearly saturated with water before the mites were added to maintain high humidity levels for the duration of the experiment. No water was added afterwards to prevent the mites from suddenly gaining mass from drinking water; food was added for some males, dependent on the treatment, (see Experimental setup). To allow air exchange, the caps of the tubes were punctured. Mites were prevented from escaping by covering the open end of the tubes with a fine mesh, kept in place by the cap. Stock cultures and mites in tubes were kept in an unlit incubator at $25^{\circ} \mathrm{C}$ and $>70 \%$ relative humidity.

\section{Experimental setup}

The experiment comprised two treatments: (1) male morph (fighter or scrambler) and (2) male nutritional history (fed or starved). The experiment was conducted over nine replicate time blocks, each replicate time block lasted 12 days and included all treatment combinations. Given that female bulb mites produce a large proportion of their eggs shortly after maturing (Gerson et al. 1983), we expected that such a period should be long enough to provide a good indication of female fecundity. Each block started 1 week after the start of the previous block.

On the first day of each block, 75 quiescent tritonymphs (the mites' final moulting stage before maturation) were collected from a stock culture and kept individually in tubes. On day 2, mites had matured and moults were removed so the moults were not consumed. Sex and male morph were determined, after which each mite was weighed individually to the nearest $0.1 \mu \mathrm{g}$ using a Sartorius Supermicro S4 electronic ultramicro balance (accuracy $\pm<0.2 \mu \mathrm{g}$, Sartorius AG, Göttingen, Germany). Hereafter, half of the scramblers and half of the fighters were given ad libitum food (one or two yeast granules, depending on granule size) between day 2 and day 3, the remainder of the males and all females were left unfed. The 'nutritional history' of the males with food will be referred to as 'fed', the nutritional history of the unfed males will be referred to as 'starved'. On day 3, all mites were weighed again. After that, females were returned to their individual tubes and paired with a male. To minimise the age difference between the two mites, each female was paired with the next male to have been collected in their quiescent tritonymph stage on day 1 . We were unaware at the time that females found in the proximity of (and subsequently paired to) scramblers in the stock cultures were generally lighter than females 
found near fighters (Online Appendix Fig. A1). Similarly, solitary (i.e. unpaired) females were the lightest group of females (Online Appendix Fig. A1). We are unsure about the cause of this discrepancy but accounted for these differences by including the female mass as a covariate in each statistical analysis (see Statistical analyses). All mites were weighed in the same order each day to keep time intervals between consecutive weighing moments similar between individuals. Females for whom no mate was available, were also returned to their tubes and remained solitary throughout the experiment and were weighed like the other females (for results, see Online Appendix Table A1). Henceforth, we weighed and checked whether each mite was alive on day 4, 5 and 12. The number of eggs laid by each female was counted on day 12. If a mite was unresponsive to the touch of a brush and did not move when turned on its dorsal side, it was considered dead. A distinction was made between three causes of death: 'natural causes', 'killed' and 'other'. Mites that died of natural causes were not deflated and may have died of an illness, mould, starvation or dehydration. Dehydrated or starved mites were recognized by two large dorsal indents on either side over the anteroposterior axis, see Fig. 1a. Killed mites were recognized by their deflated state, often as a result of puncturing and possibly cannibalism or parasitism, see Fig. 1b. Mites that were crushed or lost due to handling errors were classified as 'other'.

Fig. 1 a Starved or dehydrated adult female with two large dorsal indents on either side of the anteroposterior axis (arrows) and b fighter male (white arrow) after killing and cannibalising an adult female (black arrow)
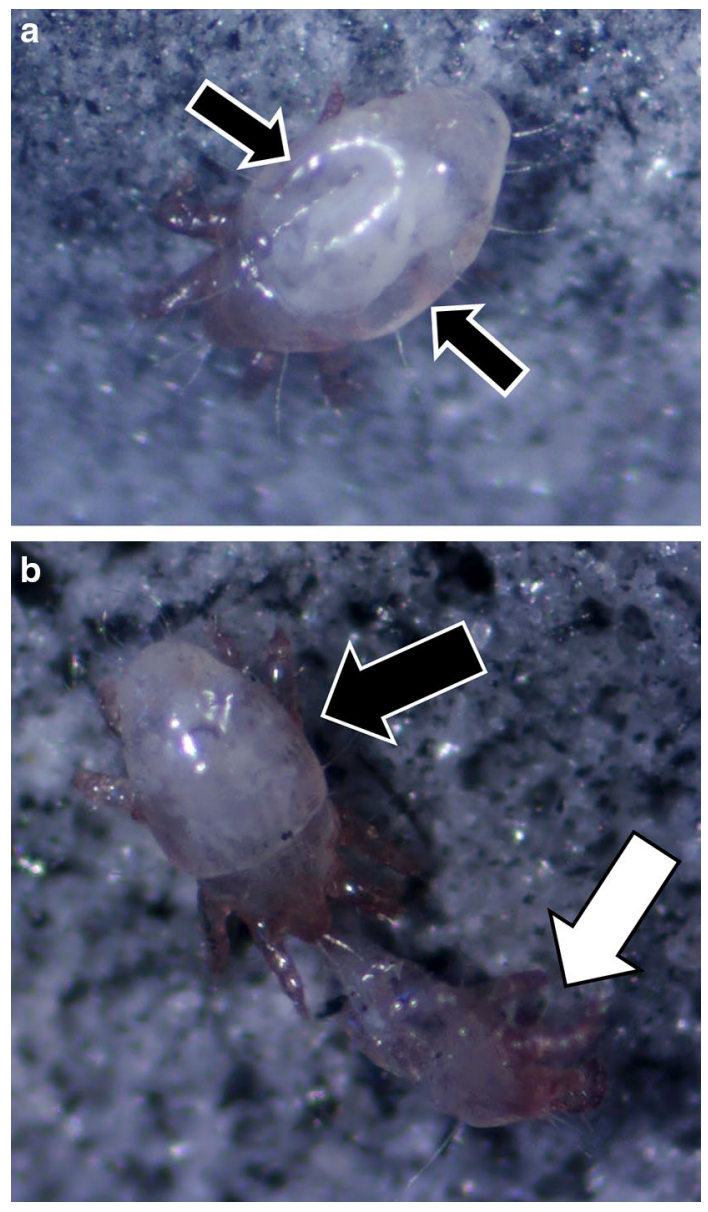


\section{Statistical analyses}

We analysed four response variables (Table 1): (1) the total number of offspring produced during the experiment (for results and replicate numbers, see Online Appendix Table A2), the mites' masses on day 4, 5 and 12 minus their mass on day 3 (on this day the mites were coupled after weighing), henceforth referred to as 'standardized mass', of (2) males (Online Appendix Table A3) and (3) females (Online Appendix Table A1) and, as fighters can kill conspecifics (Radwan et al. 2000), we analysed (4) killing incidence (binomial, 0: alive; 1: killed) (Online Appendix Table A4). We analysed the standardized mass changes of mites rather than changes in the fraction of mass or absolute mass because, if a compound is transferred from the male to the female to increase her fecundity, it is likely that the absolute mass of this compound is important to the female's fecundity rather than the mass of the compound relative to the mass of the male. Furthermore, using standardized mass changes allow for an easier comparison of potential mass flows from males to females given that they are of different initial masses because of treatments or sex (see Online Appendix Fig. A1 and Fig. A2 respectively for the absolute masses of females and males per treatment from day 2 to 12 ). The two treatments were included in the models as fixed factors: male morph (M: fighter or scrambler) and nutritional history ( $\mathrm{N}$ : fed or starved).

We tested for any effects of time on the changes in male and female mass within each replicate block by including 'time' in days (T) as a continuous factor. For the analyses of the male and female mass changes we used a three-way interaction between male morph, nutritional history and time (M.N.T). The fecundity and killing incidence analyses included a two-way interaction between male morph and nutritional history $(\mathrm{M} \cdot \mathrm{N})$; these response variables were only checked at the end of the block; hence it was not possible to test for interactions with time. To control for initial differences in mass between individuals, all four analyses included the initial male mass $\left(\mathrm{I}_{\mathrm{M}}\right)$ and initial female mass $\left(\mathrm{I}_{\mathrm{F}}\right)$ of each mating pair, measured on the day the pairs were formed (day 3), as covariates. In total, 383 replicates were started of which were 205 solitary females and 178 couples. Of these replicates 166 solitary females and 115 couples survived the full experiment.

We tested the effect of male nutritional history and male morph, and their interaction on the number of offspring produced by females mated to these males using a Generalized Linear Model (GLM) with Poisson errors. Here, only data from couples of which both individuals survived until the end of the replicate time block were used. Next, the

Table 1 Structures of the full models used to analyse the response variables of the experiment

\begin{tabular}{llll}
\hline Response variable & Model type & Error structure & Full model \\
\hline 1. Reproductive output & GLM & Poisson & $\mathrm{M} \cdot \mathrm{N}+\mathrm{I}_{\mathrm{M}}+\mathrm{I}_{\mathrm{F}}$ \\
2. Standardized male mass ${ }^{\mathrm{a}}$ & GLMM & Normal & $\mathrm{M} \cdot \mathrm{N} \cdot \mathrm{T}+\mathrm{I}_{\mathrm{M}}+\mathrm{I}_{\mathrm{F}}$ \\
3. Standardized female mass & GLMM & Normal & $\mathrm{M} \cdot \mathrm{N} \cdot \mathrm{T}+\mathrm{I}_{\mathrm{M}}+\mathrm{I}_{\mathrm{F}}$ \\
4. Killing probability & GLM & Binomial & $\mathrm{M} \cdot \mathrm{N}+\mathrm{I}_{\mathrm{M}}+\mathrm{I}_{\mathrm{F}}$ \\
\hline
\end{tabular}

Explanatory variables: male morph $(\mathrm{M})$, male nutritional history $(\mathrm{N})$, time after isolation of mites from the population in days (T). Covariates: initial male and female mass ( $\mathrm{I}_{\mathrm{M}}$ and $\mathrm{I}_{\mathrm{F}}$ respectively), i.e. the male or female mass on day 3. Model types are Generalized Linear Mixed models (GLMMs) or Generalized Linear Models (GLMs)

${ }^{\text {a }}$ The identity of the couple as well as the replicate number were used as a random factor. These random factors were not removed in the model simplification procedure 
effect of male nutritional history, male morph and time, and their three-way interaction on changes in standardized male mass were assessed using a Generalized Linear Mixed Model (GLMM) with normal errors and with block and mite-couple ID as random factors. Only data obtained during the time when both mites in a couple were alive were used. In the third analysis we tested for the effect of male nutritional history, male morph and time, and their three-way interaction on changes in the standardized female mass using a GLMM with normally distributed errors. The block and mite-couple ID were used as random factors. As in the previous analysis, all data used for this analysis were obtained during the time when both mites in a couple were alive. In our final analysis we tested for the effect of the two main effects male nutritional history, male morph and their interaction on the female killing incidence with use of a GLM with binomial errors. All mites that were missing or died due to handling errors or starvation were omitted from the dataset for this analysis.

A model simplification procedure was employed for each analysis. The least significant term of the highest order interaction was first removed from the fitted model to produce a reduced model. The difference in deviance between the two models was tested for by using a likelihood ratio test compared to a Chi squared distribution. If the removal of the term caused a significant increase in (the difference in) deviance $(P$ value $<0.05)$, then the term remains in the fitted model for the next step. Hence the new fitted model will be the same as the previous; if a term is removed, the reduced model will become the new fitted model (see Online Appendix Table A5). These steps were repeated until only terms remained of which the removal led to a significant increase in deviance. Random factors were never removed from the model. In the Results section we present parameter estimates (ê) of each significant term in the best-fitting, minimal model. The assumption of normally distributed errors of GLMs and GLMMs were confirmed by visually inspecting Q-Q plots. The data used for the GLMs were checked for overdispersion. Factors used in the GLMMs were checked for collinearity. All analyses were performed using RStudio version 1.0.136 (RStudio Team 2015), with R version 3.3.2 (R Core Team 2016) integrated. We used the packages 'Ime4' (Bates et al. 2015) for statistical analyses, 'ggplot2' (Wickham 2009) for making graphs and 'grid' (R Core Team 2016) for exporting figures. The datasets generated and analysed during the current study are available in the Figshare repository (https:// doi.org/10.6084/m9.figshare.5121880).

\section{Results}

\section{Reproductive output}

We first analysed how male nutritional history and male morph affected female fecundity (Table 1, Model 1). We found that females mated to fed males laid on average significantly more eggs than females mated to starved males $\left(\mathrm{N}: \chi_{1}^{2}=7.831, P=0.005, \mathrm{n}=115\right.$; mean number $\pm \mathrm{SE}$ [standard error] of offspring per female mated to respectively starved or fed males, $0.098 \pm 0.061 \mathrm{SE}$ and $0.333 \pm 0.130 \mathrm{SE}$ ) (Fig. 2 and Online Appendix Table A2). Female fecundity was not significantly affected by the interaction between male morph and nutritional state $\left(\mathrm{M} \cdot \mathrm{N}: \chi_{1}^{2}=3.215, P=0.073, \mathrm{n}=115\right)$, or by the main effects male morph (M: $\chi_{1}^{2}=0.007, P=0.931, \mathrm{n}=115$, Fig. 2), initial male mass $\left(\mathrm{I}_{\mathrm{M}}: \chi_{1}^{2}=1.491, P=0.222\right.$, $\mathrm{n}=115)$ or initial female mass $\left(\mathrm{I}_{\mathrm{F}}: \chi_{1}^{2}=1.339, P=0.247, \mathrm{n}=115\right)$. 
Fig. 2 Relationship between the mean number of eggs produced per mating couple during the full replicate block and male morph and male nutritional history. Vertical lines are standard errors

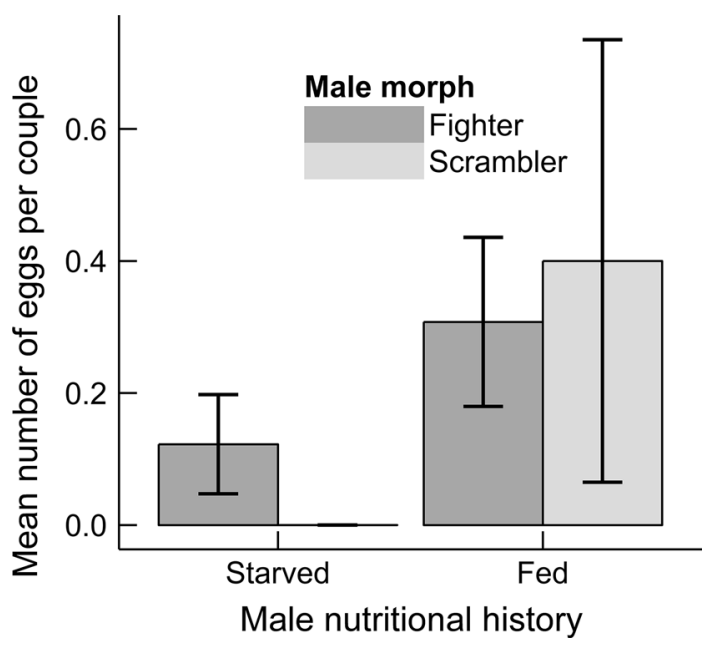

\section{Standardized male mass}

In the second analysis we assessed how male nutritional history, male morph and time affected standardized male mass (Table 1, Model 2). The results showed that the interaction between male morph and male nutritional history significantly affected standardized male mass (M.N: $\chi_{1}^{2}=4.723, P=0.030, \mathrm{n}=153$ ). Fed males lost considerably more mass than starved males (Fig. 3). Moreover, there appeared to be a large discrepancy between the difference in standardized mass change of starved scramblers and starved fighters as scramblers lost more mass than fighters (inferred from Fig. 3). This difference was much less pronounced in fed males (Fig. 3). Secondly, there was a significant negative correlation between the male mass changes and the initial male mass. Males that were relatively light on day 3 lost more mass than males that were relatively heavy on day $3\left(\mathrm{I}_{\mathrm{M}}\right.$ : $\hat{\mathrm{e}}=-0.066$, $\left.\mathrm{SE}=0.020, \mathrm{t}=-3.314, \chi_{1}^{2}=10.583, P=0.001, \mathrm{n}=153\right)$. Standardized male mass significantly decreased over time within each replicate time block $(\mathrm{T}: \hat{\mathrm{e}}=-0.028, \mathrm{SE}=0.011$,

Fig. 3 Mean change of the male standardized mass between day 12 and day 3 per male nutritional history and morph. Vertical lines are standard errors

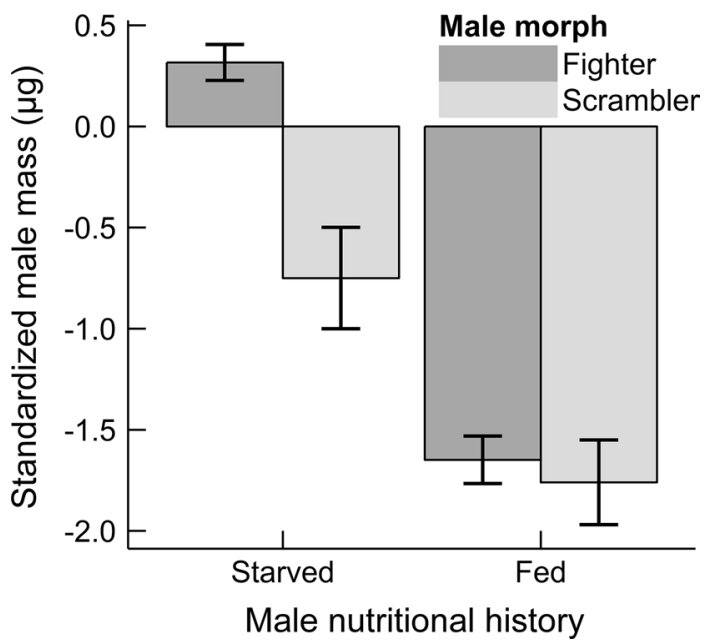


$\left.\mathrm{t}=-2.695, \chi_{1}^{2}=7.221, P=0.007, \mathrm{n}=153\right)$. Changes in standardized male mass over time were not affected by female initial mass $\left(\mathrm{I}_{\mathrm{F}}: \chi_{1}^{2}=3.671, P=0.055, \mathrm{n}=153\right)$.

\section{Standardized female mass}

In our third analysis we tested the effects of time, male nutritional history and male morph on standardized female mass (Table 1, Model 3). We found a significant effect of the interaction between male morph (M) and time (T) on standardized female mass (M-T: $\left.\chi_{1}^{2}=5.710, P=0.017, \mathrm{n}=153\right)$ : females that mated with fighters decreased in standardised mass over the course of the experiment, whereas females mated to scramblers increased in standardised mass over the course of the experiment (Fig. 4; for parameter estimates, see Online Appendix Table A6). However, on average there appeared to be no difference in female standardized mass between females mated to fighters or scramblers on day 12 (inferred from non-overlapping standard error bars in Fig. 4). Furthermore, there was no significant effect of male nutritional history $\left(\mathrm{N}: \chi_{1}^{2}=1.462, P=0.227, \mathrm{n}=153\right.$ ) or of initial female mass $\left(\mathrm{I}_{\mathrm{F}}: \chi_{1}^{2}=1.332, P=0.248, \mathrm{n}=153\right)$. There was a marginally nonsignificant trend that females mated to males with a larger initial mass increased more in mass between the start and end of the experiment $\left(\mathrm{I}_{\mathrm{M}}: \hat{\mathrm{e}}=0.057, \mathrm{SE}=0.028, \mathrm{t}=2.054\right.$, $\left.\chi_{1}^{2}=3.759, P=0.053, \mathrm{n}=153\right)$. Curiously, though to a lesser extent than mated females, solitary females increased in mass over time, despite the absence of mates, food or added water (see Online Appendix Table A1 and Online Appendix Fig. A3). We suspect that this increase in mass was due to the active uptake of ambient water vapours, as has previously been shown to occur in high humidity environments, like ours, in spiny rat mites (Echinolaelaps echidninus) (Kanungo 1963).

\section{Killing probability}

In the fourth analysis we tested the effects of male nutritional history and male morph on killing incidence (Table 1, Model 4). No females were killed by scramblers, but there were females killed by fighters (22 out of 110 ; mean \pm SE: $0.200 \pm 0.038$ ); this difference was statistically significant (M: $\mathrm{n}=137, \chi_{1}^{2}=11.419, P<0.001$, killing incidence per male morph \pm SE: fighter: 22 out of $110,0.200 \pm 0.038$ SE; scrambler: 0 out of $27,0.000 \pm 0.000$ $\mathrm{SE})$. Killing incidence was not affected by male nutritional history $\left(\mathrm{N}: \mathrm{n}=137, \chi_{1}^{2}=1.543\right.$,

Fig. 4 Mean standardized female mass over time per male morph. Female mass is calculated as a female's mass relative to its mass on day 3 , the day on which the male and female were coupled. Lines show the predicted interactive effect of male morph and time on female standardised mass: standardised female mass decreases when females are mated to fighters but increases when females are mated to scramblers. Vertical lines are standard errors

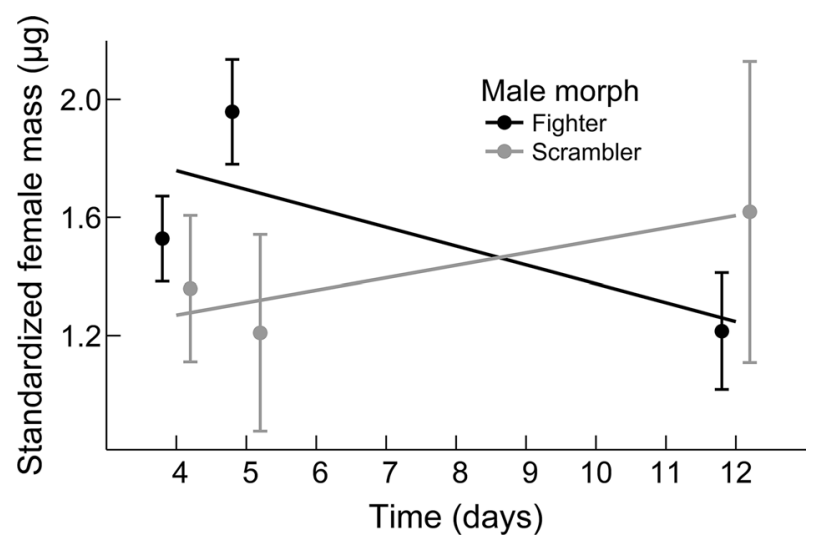


$P=0.214)$, initial male mass $\left(\mathrm{I}_{\mathrm{M}}: \chi_{1}^{2}=0.040, P=0.842, \mathrm{n}=137\right)$ or initial female mass $\left(\mathrm{I}_{\mathrm{F}}\right.$ : $\left.\chi_{1}^{2}=0.284, P=0.594, \mathrm{n}=137\right)$.

\section{Discussion}

In male-dimorphic species, one male morph, the major, typically invests resources in the development of morphological structures that are used as weapons in male-male competition (Oliveira et al. 2008). Minors, on the other hand, do not develop such structures, and therefore likely do not incur energetic costs of maintaining weaponry. As a consequence, minors might be able to invest more resources into reproduction than majors, all other things being equal (e.g. Parker 1990; Young et al. 2013). Using the male-dimorphic bulb mite $R$. robini, we investigated if females mated to a minor produced more offspring than females mated to a major, either under favourable, or unfavourable conditions. We found that, in bulb mites, female fecundity did not differ between females that were mated with a fighter (major male) or a scrambler (minor male), but that fed males sired more offspring than starved males.

Males of both morphs were either fed or starved for a single day prior to mating. Regardless of male morph, females mated to a fed male laid more eggs than those mated to a starved male. This suggests that males of both morphs invest into reproduction, but that the level of investment is dependent on their previous nutrient intake; a comparable result was found by Immonen et al. (2009) for the nuptial gift-giving Drosophila subobscura. We cannot be sure if the difference in the fecundity of females mated to fed or starved males was due to (cryptic) female preference for fed males (e.g. Fisher and Rosenthal 2006), a longer copulation time ( $R$. robini mites are known to mate for a shorter amount of time when starved, Gerson and Thorens 1982), nuptial gifts, ovipositing stimulating compounds or anything else. However, given the observation that males generally decreased in mass over the course of the experiment (Fig. 3), and their mates [compared to single females (Online Appendix Fig. A1)], increased in mass, it is plausible that some mass (and thus resource) was transferred from the male to the female during copulation.

There are alternative explanations to explain these changes in mass: males may lose mass as a result of the metabolic costs of mating as fed males mate for longer (Gerson and Thorens 1982) but consequently have a higher reproductive output. Alternatively, scramblers that are less sclerotized than fighters might be more prone to lose mass under starvation conditions (as we observed that scramblers lost more mass than fighters when starved). Females, on the other hand, may increase in mass as a result of an increased water uptake (e.g. Kanungo 1963) to accommodate the egg laying process. However, if males indeed transfer a beneficial mass to females, this could best be described as a nuptial gift. To our knowledge, this is the first reported observation suggestive of a nuptial gift transfer in mites. In Acarus siro, a species related to the bulb mite (Dabert et al. 2010), Witaliński et al. (1990) identified morphological structures in the female reproductive tract through which females could absorb nutritional contributions. If female bulb mites have similar structures, they could use these to absorb nutrients in seminal fluid (e.g. Simmons and Parker 1989) to obtain valuable substances (Bownes and Partridge 1987; Markow 1988; Edvardsson 2007; Gwynne 2008) or digest superfluous (Sivinski 1980) or non-reproductive (Silberglied et al. 1984; Swallow and Wilkinson 2002) sperm cells. To unequivocally determine the existence of nuptial gift transfer in the bulb mite, morphological studies to identify structures in the female's anatomy that take up nutrients from seminal fluid (as in 
Witaliński et al. 1990), and physiological studies that follow the fate of potential nutrients in the seminal fluid (e.g. Bownes and Partridge 1987), are required.

Contrary to our hypothesis, we found no difference in the number of offspring sired by scramblers and fighters. One explanation for the absence of a sire morph effect is that there is no difference between the two morphs in the transference of components that promote female fecundity. Alternatively, our response variable may not have been suitable to find differences between the two morphs, for example: rather than offspring number, offspring quality differed between the two male morphs (as suggested in Smallegange 2011). Furthermore, females mated to scramblers may have laid more eggs during the initial stages of the experiment but drew level with females mated with fighters towards the end of the replicate block-such a notion is supported by the results in Fig. 4 if the decreases in female mass correlate to ovipositing. As the number of eggs were only counted at the end of each replicate block, we are unable to link changes in female mass to egg laying dates.

Given that we did not find a difference in the fecundity of females mated to a scrambler or a fighter, and given that there is no evidence that scramblers perform better in sperm competitions (Radwan 1997; Radwan and Bogacz 2000), the answer to the question of why the two male morphs of the bulb mite coexist in single populations still eludes us. Skrzynecka and Radwan (2016) recently proposed that the maintenance of the dimorphism in bulb mite populations is the result of balancing selection acting on a genetic polymorphism. However, environmental conditions play a significant role in male morph expression in the bulb mite (Smallegange 2011); hence we feel this male dimorphism is best classified as a conditional strategy (Tomkins and Hazel 2007). Smallegange and Coulson (2011) estimated the heritability for the scrambler morph to be 0.41 and for the fighter morph 0.30, suggesting that this male morph expression is a polygenic trait that is both under environmental and genetic control. The challenge therefore still exists to unravel the mechanisms that maintain the conditional expression of male morphs in the bulb mite. An answer may be found in differences between the male morphs in reproductive output or life-history traits (such as maturation time Smallegange and Johansson 2014).

We found that fighters in our experiment, unlike scramblers, regularly killed their mate, regardless of their nutritional state. In another male-dimorphic mite, Sancassania berlesei, Łukasik (2010) also found that fighters killed other mites, including juveniles and females, but mostly under low food conditions. The fact that the killing incidence in $R$. robini fighters in our experiment was not affected by their nutritional state, suggests that fighters killed out of aggression or to avoid potential competition for resources available in the future (e.g. Polis 1981), rather than to obtain resources via cannibalism. It is important to find out why fighters kill conspecifics, as such interactions can greatly influence the dynamics of populations (Smallegange et al. 2018). Smallegange et al. (2018), for example, found that when fighter bulb mites were removed from exponentially growing populations (where individuals had ad libitum access to food), more fighters survived as killing pressure was reduced, so that fighter abundance actually increased. Interestingly, under food-limited, densitydependent circumstances, the harvesting of fighters from closed populations resulted in an evolutionary shift towards reduced fighter expression. Such contrasting plastic and evolutionary responses in alternative phenotype expression to variation in the strength of density-dependence complicate the unravelling of the ecological and evolutionary drivers of phenotype expression (e.g. Smallegange and Deere 2014). Interactions like these within an eco-evolutionary feedback loop, whereby population dynamics and phenotype expression interact, are likely widespread across taxa because (1) changes in population size and structure depend on differences in life history trajectories between individuals, which are a product of natural selection, and (2) the outcome and rate of trait evolution can be altered 
by population density, composition and growth. However, few studies have teased apart the drivers of the two-way interaction between trait expression and population dynamics (but see Turcotte et al. 2013; Farkas and Montejo-Kovacevich 2014). We are only now beginning to unravel the intricate relationship between male morph expression and the effects that majors and minors have on the dynamics of populations (Smallegange and Deere 2014; Smallegange et al. 2018).

In addition to the aforementioned biotic factors, abiotic factors can play a role in the maintenance of male dimorphisms as well. For example, Tomkins et al. (2011) found in the male-dimorphic bulb mite $R$. echinopus that scramblers have a selective advantage over fighters in complex habitats, as scramblers were quicker to find females than fighters were. The authors hypothesized that this difference is the result of impaired locomotion due to the enlarged leg pair in fighters (Tomkins et al. 2011). When male weaponry impairs locomotion (e.g. Moczek and Emlen 2000; Basolo and Alcaraz 2003; Wilson et al. 2009), habitat complexity and spatial variation in food availability could reduce the major males' encounter rate with both females and food, and, consequently, increase selection against expression of the major morph. Furthermore, because minor males are not impaired in their locomotion (e.g. Basolo and Alcaraz 2003; Allen and Levinton 2007; Goyens et al. 2015), they can obtain larger energy reserves than major males in complex habitats. Females have previously been found to prefer well-fed males over starved males as mates (e.g. Droney 1996; Rantala et al. 2003; Fisher and Rosenthal 2006; Albo et al. 2012); similarly, females may evolve to prefer minor males with large reserves if such reserves benefit a female's fecundity (such benefits were shown here). Male morph coexistence may then be the result of stabilising selection on whichever morph is able to achieve the largest reserves, depending on variability in environmental conditions.

In conclusion, the results of this study suggest that nuptial gifts, killing risks, and possible associated selective forces on male phenotype through potential female mate choice, complicates the understanding of the maintenance of male dimorphisms in single populations (e.g. Sinervo et al. 2000). Future research should consider incorporating populationlevel feedbacks on male morph expression, as well as the role of abiotic factors in the mating arena.

Acknowledgements We thank Jacques Deere, Flor Rhebergen and the Population Biology and Theoretical Ecology group for their useful feedback during discussion of the results. We also thank Peter de Ruiter for comments. Finally, we are grateful to the associate editor and two anonymous reviewers whose suggestions helped to improve the manuscript. IMS is funded by a MEERVOUD Grant No. 836.13.001 and VIDI Grant No. 864.13.005 from the Netherlands Organisation for Scientific Research.

\section{Compliance with ethical standards}

Conflict of interest The authors declare that they have no conflict of interest.

Open Access This article is distributed under the terms of the Creative Commons Attribution 4.0 International License (http://creativecommons.org/licenses/by/4.0/), which permits unrestricted use, distribution, and reproduction in any medium, provided you give appropriate credit to the original author(s) and the source, provide a link to the Creative Commons license, and indicate if changes were made. 


\section{References}

Albo MJ, Toft S, Bilde T (2012) Female spiders ignore condition-dependent information from nuptial gift wrapping when choosing mates. Anim Behav 84:907-912

Allen BJ, Levinton JS (2007) Costs of bearing a sexually selected ornamental weapon in a fiddler crab. Funct Ecol 21:154-161

Basolo AL, Alcaraz G (2003) The turn of the sword: length increases male swimming costs in swordtails. Proc R Soc B Biol Sci 270:1631-1636

Bates D, Mächler M, Bolker B, Walker S (2015) Fitting linear mixed-effects models using lme4. J Stat Softw 67:1-48

Bownes M, Partridge L (1987) Transfer of molecules from ejaculate to females in Drosophila melanogaster and D. pseudoobscura. J Insect Physiol 33:941-947

Burris ZP, Dam HG (2015) Spermatophore production as a function of food abundance and age in the calanoid copepods, Acartia tonsa and Acartia hudsonica. Mar Biol 162:841-853

Cordero C (1995) Ejaculate substances that affect female insect reproductive physiology and behavior: honest or arbitrary traits? J Theor Biol 174:453-461

Dabert M, Witaliński W, Kazmierski A et al (2010) Molecular phylogeny of acariform mites (Acari, Arachnida): strong conflict between phylogenetic signal and long-branch attraction artifacts. Mol Phylogenet Evol 56:222-241

Dawkins R (1980) Good strategy or evolutionarily stable strategy. In: Barlow GW, Silverberg J (eds) Sociobiology: beyond nature/nurture?. Westview Press, Boulder, pp 331-337

Díaz A, Okabe K, Eckenrode CJ et al (2000) Biology, ecology, and management of the bulb mites of the genus Rhizoglyphus (Acari: Acaridae). Exp Appl Acarol 24:85-113

Droney DC (1996) Environmental influences on male courtship and implications for female choice in a lekking Hawaiian Drosophila. Anim Behav 51:821-830

Droney DC (1998) The influence of the nutritional content of the adult male diet on testis mass, body condition and courtship vigour in a Hawaiian Drosophila. Funct Ecol 12:920-928

Dunn JC, Halenar LB, Davies TG et al (2015) Evolutionary trade-off between vocal tract and testes dimensions in howler monkeys. Curr Biol 25:2839-2844

Eberhard WG (1982) Beetle horn dimorphism: making the best of a bad lot. Am Nat 119:420-426

Edvardsson M (2007) Female Callosobruchus maculatus mate when they are thirsty: resource-rich ejaculates as mating effort in a beetle. Anim Behav 74:183-188

Eggert A, Sakaluk SK (1994) Sexual cannibalism and its relation to male mating success in sagebrush crickets, Cyphoderris strepitans (Haglidae: Orthoptera). Anim Behav 47:1171-1177

Eisner T, Smedley SR, Young DK et al (1996) Chemical basis of courtship in a beetle (Neopyrochroa flabellata): cantharidin as 'nuptial gift'. Proc Natl Acad Sci USA 93:6499-6503

Emlen DJ (2008) The evolution of animal weapons. Annu Rev Ecol Evol Syst 39:387-413

Farkas TE, Montejo-Kovacevich G (2014) Density-dependent selection closes an eco-evolutionary feedback loop in the stick insect Timema cristinae. Biol Lett 10:20140896

Fisher HS, Rosenthal GG (2006) Female swordtail fish use chemical cues to select well-fed mates. Anim Behav 72:721-725

Gerson U, Thorens D (1982) Mating frequency as an indication of food quality for the bulb mite, Rhizoglyphus robini Claparède. Int J Invertebr Reprod 5:201-206

Gerson U, Capua S, Thorens D (1983) Life history and life tables of Rhizoglyphus robini Claparède (Acari: Astigmata: Acaridae). Acarologia 24:439-448

Goyens J, Dirckx J, Aerts P (2015) Costly sexual dimorphism in Cyclommatus metallifer stag beetles. Funct Ecol 29:35-43

Gross MR (1996) Alternative reproductive strategies and tactics: diversity within sexes. Trends Ecol Evol 11:92-98

Gwynne DT (2008) Sexual conflict over nuptial gifts in insects. Annu Rev Entomol 53:83-101

Immonen E, Hoikkala A, Kazem AJN, Ritchie MG (2009) When are vomiting males attractive? Sexual selection on condition-dependent nuptial feeding in Drosophila subobscura. Behav Ecol 20:289-295

Iwata Y, Shaw PW, Fujiwara E et al (2011) Why small males have big sperm: dimorphic squid sperm linked to alternative mating behaviours. BMC Evol Biol 11:236

Jia Z, Jiang Z, Sakaluk SK (2000) Nutritional condition influences investment by male katydids in nuptial food gifts. Ecol Entomol 25:115-118

Jukema J, Piersma T (2006) Permanent female mimics in a lekking shorebird. Biol Lett 2:161-164

Kanungo K (1963) Effect of low oxygen tensions on the uptake of water by dehydrated females of the spiny rat-mite Echinolaelaps echidninus. Exp Parasitol 14:263-268 
Kooijman SALM, Metz JAJ (1983) On the dynamics of chemically stressed populations: the deduction of population consequences from effects on individuals. Hydrobiol Bull 17:88-89

Kvarnemo C, Svensson O, Manson W (2010) Investment in testes, sperm-duct glands and lipid reserves differs between male morphs but not between early and late breeding season in Pomatoschistus minutus. J Fish Biol 76:1609-1625

Locatello L, Pilastro A, Deana R et al (2007) Variation pattern of sperm quality traits in two gobies with alternative mating tactics. Funct Ecol 21:975-981

Łukasik P (2010) Trophic dimorphism in alternative male reproductive morphs of the acarid mite Sancassania berlesei. Behav Ecol 21:270-274

Markow TA (1988) Drosophila males provide a material contribution to offspring sired by other males. Funct Ecol 2:77

Markow TA, Gallagher PD, Krebs RA (1990) Ejaculate-derived nutritional contribution and female reproductive success in Drosophila mojavensis (Patterson and Crow). Funct Ecol 4:67

Moczek AP, Emlen DJ (2000) Male horn dimorphism in the scarab beetle, Onthophagus taurus: do alternative reproductive tactics favour alternative phenotypes? Anim Behav 59:459-466

Morasse S, Guderley H, Dodson JJ (2014) Paternal reproductive strategy influences metabolic capacities and muscle development of Atlantic salmon (Salmo salar L.) embryos. Physiol Biochem Zool 81:402-413

Oliveira RF, Taborsky M, Brockmann HJ (2008) Alternative reproductive tactics: an integrative approach, 1st edn. Cambridge University Press, Cambridge

Parker GA (1983) Arms races in evolution—an ESS to the opponent-independent costs game. J Theor Biol 101:619-648

Parker GA (1990) Sperm competition games: raffles and roles. Proc R Soc Lond B 242:120-126

Polis GA (1981) The evolution and dynamics of intraspecific predation. Annu Rev Ecol Syst 12:225-251

Prenter J, MacNeil C, Elwood RW (2006) Sexual cannibalism and mate choice. Anim Behav 71:481-490

R Core Team (2016) R: A language and environment for statistical computing. R Foundation for Statistical Computing, Vienna, Austria. https://www.R-project.org/

Radwan JW (1995) Male morph determination in two species of acarid mites. Heredity 74:669-673

Radwan JW (1997) Sperm precedence in the bulb mite, Rhizoglyphus robini: context-dependent variation. Ethol Ecol Evol 9:373-383

Radwan JW, Bogacz I (2000) Comparison of life-history traits of the two male morphs of the bulb mite, Rhizoglyphus robini. Exp Appl Acarol 24:115-121

Radwan JW, Czyż M, Konior M, Kołodziejczyk M (2000) Aggressiveness in two male morphs of the bulb mite Rhizoglyphus robini. Ethology 106:53-62

Rantala MJ, Kortet R, Kotiaho JS et al (2003) Condition dependence of pheromones and immune function in the grain beetle Tenebrio molitor. Funct Ecol 17:534-540

Roff DA (1996) The evolution of threshold traits in animals. Q Rev Biol 71:3-35

RStudio Team (2015) RStudio: integrated development for R. RStudio, Inc., Boston, MA. http://www.rstud io.com/

Silberglied RE, Shepherd JG, Lou DJ (1984) Eunuchs: the role of apyrene sperm in Lepidoptera? Am Nat 123:255-265

Simmons LW, Parker GA (1989) Nuptial feeding in insects: mating effort versus paternal investment. Ethology $81: 332-343$

Simmons LW, Tomkins JL, Hunt J (1999) Sperm competition games played by dimorphic male beetles. Proc R Soc B Biol Sci 266:145-150

Simmons LW, Emlen DJ, Tomkins JL (2007) Sperm competition games between sneaks and guards: a comparative analysis using dimorphic male beetles. Evolution 61:2684-2692

Sinervo BR, Svensson EI, Comendant T (2000) Density cycles and an offspring quantity and quality game driven by natural selection. Nature 406:985-988

Sinervo BR, Bleay C, Adamopoulou C (2001) Social causes of correlational selection and the resolution of a heritable throat color polymorphism in a lizard. Evolution 55:2040-2052

Sivinski J (1980) Sexual selection and insect sperm. Florida Entomol 63:99-111

Skrzynecka AM, Radwan JW (2016) Experimental evolution reveals balancing selection underlying coexistence of alternative male reproductive phenotypes. Evolution 70:2611-2615

Smallegange IM (2011) Complex environmental effects on the expression of alternative reproductive phenotypes in the bulb mite. Evol Ecol 25:857-873

Smallegange IM, Coulson T (2011) The stochastic demography of two coexisting male morphs. Ecology 92:755-764 
Smallegange IM, Deere JA (2014) Eco-evolutionary interactions as a consequence of selection on a secondary sexual trait. In: Moya-Laraño J, Rowntree J, Woodward G (eds) Advances in ecological research. Academic Press, Oxford, pp 145-169

Smallegange IM, Johansson J (2014) Life-history differences favor evolution of male dimorphism in competitive games. Am Nat 183:188-198

Smallegange IM, Fernandes RE, Croll JC (2018) Population consequences of individual heterogeneity in life histories: overcompensation in response to harvesting of alternative reproductive tactics. Oikos. https://doi.org/10.1111/oik.04130

Smith JM (1982) Evolution and the theory of games. Cambridge University Press, Cambridge

Smith CC, Ryan MJ (2010) Evolution of sperm quality but not quantity in the internally fertilized fish Xiphophorus nigrensis. J Evol Biol 23:1759-1771

Stuglik MT, Babik W, Prokop ZM, Radwan JW (2014) Alternative reproductive tactics and sex-biased gene expression: the study of the bulb mite transcriptome. Ecol Evol 4:623-632

Swallow JG, Wilkinson GS (2002) The long and short of sperm polymorphisms in insects. Biol Rev Camb Philos Soc 77:153-182

Taborsky M (1997) Bourgeois and parasitic tactics: do we need collective, functional terms for alternative reproductive behaviours? Behav Ecol Sociobiol 41:361-362

Taborsky M, Oliveira RF, Brockmann HJ (2008) The evolution of alternative reproductive tactics: concepts and questions. In: Oliveira RF, Taborsky M, Brockmann HJ (eds) alternative reproductive tactics. Cambridge University Press, Cambridge, pp 1-22

Tomkins JL, Hazel WN (2007) The status of the conditional evolutionarily stable strategy. Trends Ecol Evol 22:522-528

Tomkins JL, Hazel WN, Penrose MA et al (2011) Habitat complexity drives experimental evolution of a conditionally expressed secondary sexual trait. Curr Biol 21:569-573

Turcotte MM, Reznick DN, Daniel Hare J (2013) Experimental test of an eco-evolutionary dynamic feedback loop between evolution and population density in the green peach aphid. Am Nat 181:S46-S57

Vahed K (1998) The function of nuptial feeding in insects: a review of empirical studies. Biol Rev 73:43-78

Wickham H (2009) ggplot2: Elegant graphics for data analysis. Springer, New York

Wilson RS, James RS, Bywater C, Seebacher F (2009) Costs and benefits of increased weapon size differ between sexes of the slender crayfish, Cherax dispar. J Exp Biol 212:853-858

Witaliński W, Szlendak E, Boczek J (1990) Anatomy and ultrastructure of the reproductive systems of Acarus siro (Acari: Acaridae). Exp Appl Acarol 10:1-31

Young B, Conti DV, Dean MD (2013) Sneaker 'jack' males outcompete dominant 'hooknose' males under sperm competition in Chinook salmon (Oncorhynchus tshawytscha). Ecol Evol 3:4987-4997 